Int. J. Speleol., 26, 3-4 (1997): 3 - 19

\title{
LES DEUX FACES DE LA PENSÉE DE E.A. MARTEL
}

\author{
J. Choppy *
}

NOTA. Sous le titre "Deux aspects de la pensée de Martel", une première version de ce texte fut publié dans le mémoire ${ }^{\circ} 7 / 1982$ du Spéléo-club de Paris (pages 35-47) consacré à "L'origine des cavernes calcaires" de W.M. Davis; je remercie le Spéléo-club de Paris d'avoir autorisé ce remodelage.

Réunion de textes de Martel:

\section{RESUMÉ}

1 - Extraits du Nouveau Traité des Eaux Souterraines (1921), donnant l'essentiel de la pensée de l'auteur sur l'hydraulique et la morphologie du karst.

2 - En opposition à cette vision, rappelée de nombreuses fois par l'auteur, extraits de diverses publications (de 1894 à 1930), admetant qu'un fonctionnement en régime noyé était possible: Martel est donc l'un des tout premiers ayant observé des formes de creusement en régime noyé, et les ayant interpretées comme telles.

\section{INTRODUCTION}

À notre époque, les conceptions traditionnelles sur le creusement karstique sont de plus en plus contestées; elles sont progressivement remplacées par une vision nouvelle tenant compte d'informations beaucoup plus abondantes et variées. Il est sans doute important de faire le point des théories antérieures, qui risquent d'apparaître bientôt tellement obsolètes qu'on ne fera plus l'effort de les consulter... se privant ainsi de nombreuses idées qui gardent leur part d'intérêt.

Pour présenter les grandes lignes des idées de Martel, mon choix s'est d'abord porté sur une démonstration tirée du Nouveau traité des eaux souterraines, dépouillée des innombrables incidentes qui rendent le fil des idées difficile à suivre dans le texte original, mais en conservant le plan de celui-ci (les notes infra-paginales ont été complétées).

Toutefois la pensée de Martel n'est pas aussi monolithique qu'on le croit d'ordinaire : des textes d'époques différentes, depuis Les Abîmes en 1894, jusqu'à La France ignorée en 1930, en sont la preuve.

Car ces extraits rendent un son inhabituel sous la plume de Martel : Ils montrent qu'en filigrane de son discours "fluviatile", un autre courant d'idée n'a jamais cessé d'être présent dans son esprit, selon lequel un creusement en régime noyé, avec une morphologie particulière, pouvait exister. Certes, il ne s'agit pas d'une théorie cohérente, et l'entraînement verbal provoque des contradictions (que je n'ai pas soulignées dans ces extraits) entre les stéréotypes de l'auteur et des observations contraires.

Mais l'on se prend à rêver aux théories qu'aurait défendues Martel s'il avait, par exemple, fait des explorations de plus longue durée dans les Iles Britanniques. Et, s'il n'est pas question de faire de Martel un précurseur de Bretz, on doit reconnaître qu'il fut l'un des tout premiers ayant observé des formes de creusement en régime noyé, et les ayant interprétées comme telles. 


\section{1\% Les idées fluviatiles}

\section{NOUVEAU TRAITÉ DES EAUX SOUTERRAINES}

Doin éd. Paris, 1921, pages 218-324

\section{"INEXISTENCE DU GRAND RÉSERVOIR ET DES NAPPES D'EAU DANS LES CALCAIRES (218-222)}

Quand les percolations ont disparu dans les sols calcaires, par les différentes sortes de points d'absorption, que deviennent-elles à l'intérieur ?...

Y a-t-il, dans les calcaires, accumulation de l'eau souterraine en grands réservoirs très amples et très creux, - extension en larges nappes -, ou descente par tuyaux et circulation par véritables rivières?

Les nombreux cours d'eau souterrains, - absorbés tout faits ou constitués dans le sol par concentration interne des percolations -, actifs (encore vivants), desséchés (déjà morts) ou intermittents (ne fonctionnant qu'après les pluies), que l'on a pu effectivement découvrir et suivre, parfois sur des kilomètres d'étendue,... ont montré leur indiscutable ressemblance (courants, confluents, lacs, rapides, cascades, crues, etc.) avec les rivières extérieures.

Ils en diffèrent cependant par l'existence de trois obstacles spéciaux : $1^{\circ}$ ) les rétrécissements extrêmes des galeries qui, parfois, ne mesurent plus que quelques centimètres de largeur; - $2^{\circ}$ ) les éboulements intérieurs constituant des barrages que les eaux doivent traverser ou contourner (1); et surtout, les abaissements de plafonds qui provoquent l'immersion totale de la roche encaissante, en voûtes mouillantes ou siphons d'aqueducs.

Ces dispositifs ont prouvé... l'inexistence de véritables nappes d'eau dans le calcaire...

Il est définitivement établi que le dernier terme de la circulation souterraine, en terrains grandement fissurés (calcaires et craies), est celui de la réapparition des eaux, sous forme de puissantes et capricieuses résurgences...

Notons seulement... que ces points d'émergence sont tantôt impénétrables à l'homme, tantôt... ouverts en vastes cavernes où l'on a pu, plus ou moins loin, remonter le fil de l'eau à l'intérieur du sol..."

\section{“LES RÉSEAUX HYDROGRAPHIQUES SOUTERRAINS (226-228)}

Le géologue portugais Delgado (2) écrivait : "Les grottes de Cesareda... sont dans des calcaires... dont les fissures absorbent les pluies. À la base des calcaires, la fontaine d'0lha-Marinho grossit abondamment pendant la saison pluvieuse... L'intérieur de

1) Certains courants torrentiels sont encombrés aussi d'éboulis sous lesquels ils disparaissent plus ou moins longtemps.

(2) DELGADO J.F.N. - 1867, Noticia acerca das grutas da Cesareda. Commission Géologique du Portugal, Lisbonne. 
Césaréda doit recéler un vaste réseau de couloirs souterrains, communiquant entre eux; et plusieurs s'ouvrent à la superficie du sol par des entonnoirs où les eaux pluviales sont reçues et s'infiltrent". Entonnoirs d'absorption, réseaux souterrains, réapparitions d'eaux sont donc ici réunis et déjà observés très exactement.

De même Duponchel (3)... avait singulièrement vu juste... :

"Les failles aquifères sont, en général, recoupées par d'autres, produisant une série de réseaux souterrains, en communication plus ou moins directe les uns avec les autres."

Il convient d'y ajouter que les subdivisions de ce réseau sont accidentées d'obstacles..., chutes brusques, poches d'eau, réservoirs, etc..., des plus capricieux, communiquant souvent entre eux par des anastomoses... très étendues, tandis qu'ailleurs plusieurs portions du réseau peuvent être complètement indépendantes les unes des autres; il en résulte des dénivellations importantes, des réservoirs souterrains, des irrégularités considérables des écoulements de l'eau; beaucoup de ces irrégularités se compensent entre elles, au point d'aboutir à la pérennité, sinon à la régularité complète des émergences..."

\section{"SIMILITUDE DES COURANTS SOUTERRAINS AVEC CEUX DE LA SURFA- CE (228-234)}

Les courants souterrains sont aujourd'hui reconnus dans un nombre considérable de cavernes, avec des largeurs et profondeurs qui permettent d'y effectuer de véritables navigations... Parfois, ces rivières se calment et s'élargissent dans de vastes salles de cavernes, au point de former de réels lacs, ou du moins des bassins...

Des rapides absolument torrentiels s'écroulent çà et là, parmi des blocs d'éboulis souvent monstrueux détachés des voûtes... Les cascades souterraines ont été trouvées... dans tous les pays...

On ne saurait donc plus considérer les cours d'eau souterrains comme des exceptions...

Quant aux confluents, le plus grandiose est dans la grotte de Kleinhaüsel à Planina..., sous une voûte de 20 à 30 mètres d'élévation... La grotte desséchée de Sare (BassesPyrénées)... présente même la concentration de trois adductions naturelles...

Les crues souterraines... sont des spectacles grandioses et terrifiants. On comprend devant ces phénomènes, bousculant et transportant des blocs énormes de rocs et des madriers du dehors, comment ont pu s'agrandir en immenses cavernes les lithoclases originairement étroites du sous-sol.

L'ampleur considérable, le caractère subit et le peu de durée de ces sortes de crues souterraines contredisent formellement l'hypothèse des nappes dans les calcaires: car elles se manifestent en hauteur, bien plus qu'en largeur.

Cvijic' (4), cependant... a cru devoir appeler courants ascendants les gonflements de

(3) DUPONCHEl A. - 1868, Traité d'hydraulique et de géologie agricole; Eug. Lacroix éd., Paris "Les cavernes ne sont que les anciens réservoirs des sources, dont le niveau s'est abaissé, à mesure que les vallées ont été plus profondément labourées. [Ces réservoirs] donnent une idée de ceux qui fonctionnent encore aujourd'hui à un niveau inférieur et communiquant parfois avec eux".

(4) CVIJIC' J. - 1918, Hydrographie souterraine et évolution morphologique du Karst; Recueil des travaux de l'Institut de Géographie Alpine, VI/4, 375-426. 
cette sorte que l'arrivée des pluies produit dans les cavernes du Karst, quand les eaux envahissent ainsi grottes et rivières souterraines; mais c'est qu'alors... fonctionnent... en mécanismes de trop-pleins, les anciens orifices supérieurs généralement à sec des résurgences actuelles."

\section{"ASCENSION DES EAUX SOUTERRAINES DANS LE TUYAUTAGE DES ABÎMES (234-236)}

Cvijic' n'a pas assez insisté sur l'ascension qui se produit en même temps dans les parties inférieures des... avens, où l'eau se met rapidement en charge, comme en de vrais tuyaux de cheminées. - Après les pluies, l'élévation de l'eau, dans ces abîmes, en fait violemment sortir des courants d'air chassés par la montée de l'eau... Fournet (5)... les avait appelés abîmes verticaux emissifs."

\section{“RAPIDITÉ DES MISES EN CHARGE (236)}

Ces ascensions rapides sont la meilleure des réfutations des nappes d'eau dans le calcaire, puisqu'elles témoignent de l'impossibilité d'une expansion des eaux dans le sens latéral, en largeur.

Au contraine... les gonflements d'eaux souterraines (et par conséquent d'émergences) sont lents dans les vraies nappes des terrains détritiques, parce que la surélévation générale du niveau souterrain s'étend latéralement...; s'ils sont brusques et violents pour les résurgences, c'est parce que l'accroissement de volume interne s'élève étroitement et se met en charge dans le tuyautage surtout vertical de la fissuration; alors s'établissent les conduites forcées et les anastomoses...

Ces rapides mises en charge, si caractéristiques de la circulation en terrains calcaires et crayeux, ont été souvent et matériellement constatées dans les abîmes du karst : à 10 ou 12 atmosphères au lac de la Mort, à l'extrémité de la Recca souterraine de Saint-Canzian (Istrie), à plus de 10-16 atmosphères aux gouffres de Trebiciano..."

\section{“VARIATIONS DES TROP-PLEINS ET RÉSURGENCES (237)}

Les gonflements de rivières souterraines se manifestent surtout pour les gouffres qui sont absorbants en temps normal ou au début des pluies, puis émissifs dès que celles-ci se prolongent... Ce sont les cours d'eau, les réseaux souterrains et les résurgences qui gonflent leurs trop-pleins; ceux-ci entrent en fonctions dès qu'il y a pléthore ou hypertrophie aqueuse, dans les fissures aquifères qui les alimentent."

\section{“LACS ALTERNATIFS (239-242)}

Quant aux grandes dépressions fermées ou polje, du karst dinarique ou liburnien,

(5) FOURNET J. - 1858, Hydrographie souterraine; Acad. sciences, belles-lettres et arts de Lyon, 4 mai, 221-296 
elles sont, suivant leur situation par rapport au régime actuel de l'eau dans le soussol, ou bien toujours à sec, ou bien périodiquement inondées. Dans ce dernier cas, leurs points d'absorption... $\quad$ sont alternativement émissifs ou absorbants... Un des plus curieux ensembles est celui des polje... drainés par la vallée de la Cetina en Dalmatie (fig.1)... Elle draine souterrainement une douzaine de polje... qui mesurent près du double (2.737 kilomètres carrés) de son propre bassinsubaérien (1.463 kilomètres carrés). Celui-ci est séparé des polje par l'axe dit des Alpes dinariques.... Les polje mêmes reçoivent aussi des résurgences alimentées par des absorptions dinariques...

[La] différence d'altitude de plus de 700 mètres entre les polje et la mer, serait à elle seule exclusive de la conception des nappes d'eau si le phénomène des engouffrements et reflux alternatifs ne dénonçait pas déjà la réalité du remplissage, d'ordre vertical, des fissures qui rendent les gouffres émissifs, quand elles sont bourrées d'eau à refus...

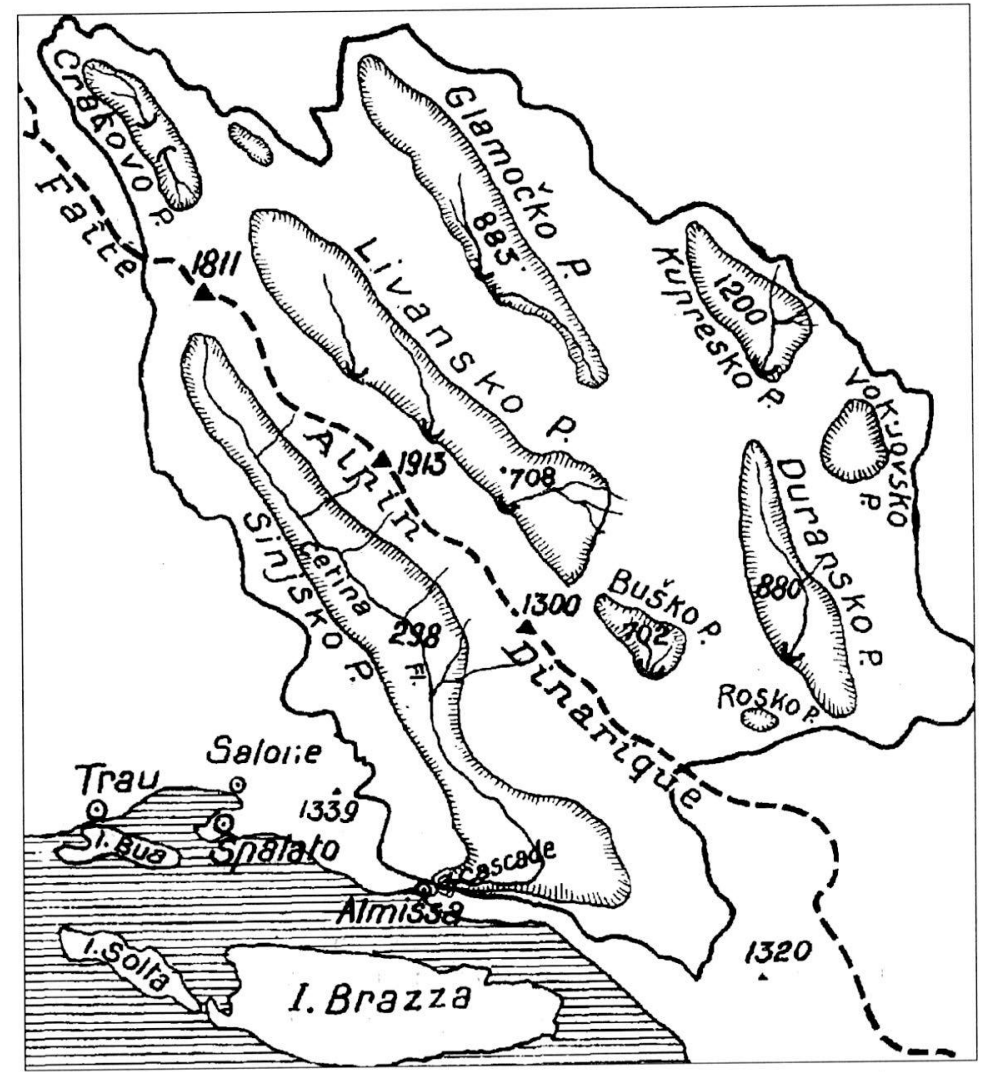

Fig. 1 : Vallée de la Cettina (Dalmatie), ouverte jusqu'à la mer, et ses polje tributaires à émissaires souterrains (Nouveau traité des eaux souterraines fig. 119 p. 200).

Dans le pays plat qu'est la Russie, il existe quantité d'entonnoirs du calcaire et du gypse, effondrés par suite du peu d'épaisseur du sol au-dessus de la circulation sou- 
terraine, notamment dans les régions d'Oufa et de Toula...

Une des plus curieuses alternances connues est celle de la Green River (Kentucky) : tantôt elle recueille comme affluents les eaux qui sortent de Mammoth-Cave, tantôt au contraire elle se déverse dans leurs galeries. selon que les pluies ont été plus abondantes, dans le bassin de la rivière ou dans les régions absorbantes qui alimentent les courants de la caverne.

Le plus étrange des cas nous vient d'Amérique :

En 1891, près de Gasneville (Floride du Nord), le lac d'Alachua, long de 16 à 24 kilomètres, n'existait plus. C'est la seconde fois depuis 1823 que le phénomène se produisait. À cette date, le lac était une grande prairie avec un petit ruisseau et un sink (6). En 1868, de grandes pluies submergèrent la prairie qui se dessécha [ensuite] rapidement. En 1873, après d'autres pluies, le sink déborda et, pendant plusieurs années, les eaux montèrent jusqu'à former un lac assez important pour avoir été parcouru pendant quinze ans par de petits vapeurs. Dès 1889 , le niveau de l'eau baissa de nouveau et, vers le milieu d'août 1891, avec une telle rapidité (environ 25 centimètres par jour) qu'au bout de quatre semaines le dessèchement était complet. Il est clair que le déblaiement des passages souterrains et inconnus du sink est la cause de ce drainage, tandis que leurs obstructions accidentelles et inexpliquées provoquaient l'inondation.

\section{“TROIS OBSTACLES A LA CIRCULATION DES RIVIÈRES SOUTERRAINES $(242-257)$}

$1^{\circ}$ ) Les rétrécissements se produisent quand la compacité locale de la roche a empêché la fissure originaire d'être élargie aussi amplement que sur d'autres points moins résistants. Il y en a qui ne laissent plus que quelques centimètres de vide, et il arrive que les dépôts de concrétion... bouchent entièrement la galerie... Alors, l'eau de la rivière passe (ou passait) sous l'obstacle et, quand surviennent les grandes pluies ou les très violents orages, il se trouve que les eaux de surface, abondamment infiltrées, n'ont plus un écoulement suffisant et que leur reflux les accumule en amont; elles se mettent donc en charge, en montant parfois très haut, et elles peuvent transformer en réservoirs temporaires de longues sections de cavernes.

Dans les rétrécissements, même non obstrués, la chasse de l'eau devient alors terrible et produit des phénomènes d'érosion analogues à ceux que l'on constate dans les cluses... des montagnes.

Aux Katavothres (7) de Grèce, les rétrécissements sont tels que les cavités intérieures se trouvent réunies par de véritables goulots. Ceux-ci s'engorgent très facilement par des bouchons de débris végétaux entraînés...

Dans le Scialet de la Cèpe (Vercors), les deux parois d'une galerie pleine d'eau (profondeur plus de trois mètres) sont rapprochées $(0 \mathrm{~m}, 30)$ au point de n'avoir pu livrer passage à l'homme... Ce réservoir inexploré est en hauteur et non en largeur...

$2^{\circ}$ ) Les éboulements intérieurs produisent de semblables effets de reflux, d'autant plus qu'ils sont souvent colmatés par les argiles de décalcification et les autres produits de l'ablation souterraine; la rivière du Tindoul-de-la-Vaissière est ainsi barrée

(6) $\operatorname{sink}=$ perte (américain)

(7) katavothre = gouffre absorbant (grec) 
vers le milieu de son cours par l'éboulis colossal du gouffre qui fait gonfler son cours d'amont à la moindre pluie...

$\left.3^{\circ}\right)$ Presque partout, dans les résurgences et rivières souterraines, on se trouve arrêté, après un parcours plus ou moins long, par des siphons naturels généralement inverses (c'est-à-dire tubés en U); ils sont formés de voûtes mouillantes, de murailles rocheuses immergées dans l'eau sur une profondeur et une épaisseur variables... Ces siphons, véritables demi-vannes, de section restreinte, régularisent dans une certaine mesure le débit des eaux souterraines, qu'ils retiennent pour partie, dans les réservoirs ou espaces libres situés en amont...

Presque toutes les rivières souterraines du calcaire se sont montrées pourvues de siphons; leurs tunnels libres de part en part sont rares. Les mises en charge hydraulique dans les cavernes à courants d'eau prouvent l'existence de canaux, et surtout de cloches étanches qu'on a du reste matériellement vues à Han, Padirac... Pour les sources intermittentes, le siphon paraît nécessaire...

À la grotte de Vrsnika (Carniole), C. Hrasky, en 1887, a pu contourner artificiellement des siphons intérieurs, et les modifier de manière à débarrasser la vallée fermée de la Racna de ses inondations périodiques...

Au gouffre de la Piuka-Jama, F. Kraus (8) en a désamorcé plusieurs, en abaissant le niveau des eaux par des travaux de mines... En outre,... après le grand éboulement du premier siphon, il y a un embranchement qui rejoint, encore en siphonnant, la dérivation de la Cerna Jama... MM. Bock et Mülhofer ont trouvé, au sud du dernier bassin de cette dérivation, une source qui remonte de 5 mètres de profondeur; elle s'écoulait alors avec $9^{\circ} \mathrm{C}$ vers le cours souterrain de la Piuka qui était à $18^{\circ} \mathrm{C}$.

Le 20 septembre 1898, en très basses eaux, j'ai pu franchir un siphon, - désamorcé sur 0 m,20 de hauteur et 0m,50 de largeur, - à la nage, à Han sur Lesse; il ne m'a mené qu'à une cloche de 10 mètres de diamètre, circulaire, sans aucune rive, avec un autre siphon amorcé (9)...

La plupart des siphonnements sont inverses ou renversés... (siphons d'aqueducs). Mais on a constaté aussi plusieurs fois l'existence matérielle de siphons normaux... avec la courbure en haut... À Poux-Blanc (Tarn-et-Garonne)... au fond, le niveau de l'eau s'y trouve, en sécheresse, plus bas que le thalweg de Poux-Nègre; quand les pluies ont rempli d'eau les fissures du plateau, la pression hydraulique les élève dans les deux puits terminaux; le véritable siphon normal que constitue la galerie du deuxième étage, s'amorce... et le trou de Poux-Blanc jaillit au dehors, parce que son orifice est plus bas que la galerie du deuxième étage...

C'est donc en amont de ces siphons que les rivières souterraines, après les pluies, peuvent se mettre en pression dite hydrostatique, sur des hauteurs parfois considérables, formant des réserves d'eau souterraines qui s'écoulent plus ou moins lentement, selon le diamètre du siphon, l'étendue du réservoir, sa pression hydraulique, etc... : ainsi se réalisent les oscillations de niveau des émergences telles que Vaucluse, la Touvre,...

Dans les grottes sèches (anciennes rivières souterraines mortes), on a rencontré aussi des abaissements de voûtes qui fonctionnèrent jadis comme siphons... Beaucoup sont aujourd'hui incrustés de stalactites qui les dentèlent en peignes... La plupart des gale-

(8) KRAUS F. - 1894, Höhlenkunde; Carl Gerold's Sohn, Wien, 308 p.; p. 92

(9) VAN DEN BROECK E. et al. 1910, Les cavernes et rivières souterraines de la Belgique; Lamertin

éd. Bruxelles, 2 tomes, 1592 p. + annexes; fig. 42 p. 77, p. 91 
ries rampantes de cavernes... sont aussi d'anciens siphons. Le petit tunnel de la grotte de Lombrive (à Ussat, Ariège) est très remarquable : la compacité de sa roche l'a empêché de s'agrandir sous la pression formidable des eaux qui s'accumulaient en amont à plus de 5 atmosphères; celle-ci, au contraire, a fini par vidanger la grotte à son autre extrémité, en transformant une fissure en un gouffre de 40 mètres de creux qui l'a réunie à la grotte de Sabart."

\section{“ORIGINE TECTONIQUE DES SIPHONS (257)}

Les siphons ont souvent une origine tectonique, quand ils sont dus à des plissements locaux de couches, ou à de longues inflexions de strates en fond de bateau; dans ces cas, l'eau, suivant le pendage général, remonte forcément par un vase communiquant, si les strates qui l'enferment sont tout à fait imperméables ou compactes, au point de ne lui offrir aucune fissure d'échappement vers des points plus bas. Il en résulte que les émergences du calcaire peuvent ramener les eaux d'un niveau inférieur à celui même où elles sourdent (90 mètres à Bournillonne, Isère) ou siphon d'Arbois...; 50 mètres au moins à Vaucluse... C'est de cette manière générale, par le jeu du tube en $\mathrm{U}$, que naissent, souvent à grandes profondeurs, des sources sous-fluviales, souslacustres et sous-marines, qui sont fréquemment de puissantes rivières..."

\section{“TUNNELS NATURELS SANS SIPHONNEMENT (259-260)}

Comme les eaux souterraines échappent, plus encore que tous les autres phénomènes naturels, aux lois absolues, on connaît un certain nombre d'exemples de rivières caverneuses, même fort longues, dont nul siphon n'interrompt le cours; mais ce sont des exceptions où l'eau absorbée peut être suivie d'un bout à l'autre, sans solution de continuité, sans tubes en U interrupteurs, dans de véritables tunnels... Ces souterrains présentent les accidents habituels aux rivières des cavernes et notamment les traces les plus nettes d'érosion..."

\section{“GALERIES ATTEINTES PAR DES ABÎMES (263-264)}

On a pu accéder aux rivières souternines, non seulement par les pertes et les résurgences non fermées en siphons, mais aussi par des abîmes ou cavernes, ouverts sur leur cours même ou sur un affluent latéral... On a rejoint de même des galeries souvent grandioses, dont les courants ont disparu ou du moins n'y circulent plus que temporairement après les grandes pluies... d'autres sont tout à fait mortes.

Les rivières souterraines ainsi révélées se sont presque toutes trouvées impraticables au bout d'un parcours plus ou moins long, par suite des trois obstacles énumérés cidessus, tantôt fentes trop étroites... tantôt éboulements ou encore des bouchons de limon faisant office d'obturateurs... tantôt, surtout, siphons..."

\section{“ABSENCE DE NAPPE D'EAU DANS LES CALCAIRES (267-269)}

Vaucluse est l'exemple le plus typique de cette erreur : "Il est généralement admis 
que Vaucluse constitue le trop-plein d'une vaste nappe d'eau souterraine" (carte géologique au 1/80 000e, feuille de Forcalquier, 1892)... En aucune manière : Vaucluse est simplement le débouché d'un puissant fleuve souterrain, tirant son origine de la percolation, par les abîmes et fentes des plateaux de Sault, Saint-Christol... et non pas l'affleurement d'une nappe; l'écoulement s'opère par de longs et hauts canaux renflés au milieu et affectés de considérables variations de niveau; ces variations proviennent : $1^{\circ}$ ) des irrégularités des précipitations atmosphériques et des infiltrations; $2^{\circ}$ ) des rétrécissements, siphonnements et éboulements intérieurs, faisant fonctions de vannes retardatrices et transformant les canaux en réservoirs temporaires étroits. Les grandes différences de pression hydraulique ainsi engendrées provoquent une mise en charge plus ou moins considérable des veines liquides ramifiées sous terre; et la répercussion de cette pression variable sur le dernier vase communiquant qui forme (peut-être dans une faille) l'émergence de Vaucluse, amène les écarts de niveau et de débit de la fontaine..."

\section{“ERREURS DES NAPPISTES (269-270)}

Il n'est plus possible de dire avec $\mathrm{O}$. Keller (10 \} et tant d'autres que, "lorsque les grottes vides se trouvent, par leur situation, en contact avec la partie supérieure d'une nappe aquifère, elles se remplissent d'eau et se vident alternativement, suivant que la nappe elle-même se gonfle ou se dégonfle sous l'action des pluies ou de la sécheresse". Cette conception du régime hydroogique souterrain des terrains à grottes, qu'Alfred Grund en somme n'a fait que répéter (11), est tout à fait contraire aux dernières données exploratrices de la science...

C'est une certitude absolue, eu égard au grand nombre de points d'absorption trouant la surface des régions calcaires, que, dans un massif de cette formation, des milliers de tuyaux de pipes forment les verticales ou obliques descentes de gouttières, qui mènent les infiltrations au réseau de canaux aquiducteurs subhorizontaux.

Toute la confusion que les nappistes se refusent à faire cesser, remonte en fait à une inadvertance de rédaction de Belgrand qui, en attestant l'existence de courants souterrains dans les fissures de roches dures, ainsi que dans les interstices de grains de sable des terrains arénacés, déclare qu' "on donne généralement à ces cours d'eau le nom de nappes souterraines" (12). Là fut l'erreur - qui persiste toujours - de dire que les nappes sont des courants; assurément, certaines nappes sont courantes, possèdant un écoulement souvent rapide; mais les ruisseaux souterrains aujourd'hui connus ne sont pas des nappes."

\section{“CONDAMNATION DES NAPPES DISCONTINUES ET DES NIVEAUX AQUIFĖRES EN TERRAINS CALCAIRES (270-271)}

$\mathrm{Au}$ fond, Belgrand faisait bien la distinction en déclarant que, dans les granites et les

(10) KELLER O. - 1897, Saturation hygrométrique de l'écorce du globe: Détermination de l'eau de carrière; Annales des Mines, 1897, pp. 32-87.

(11) GRUND A. - 1903, Die Karsthydrographie, Studien an Westbosnien; Penck's Geogr. Abhandl., Leipzig, VII/3, 103-200

(12) BELGRAND - 1872, La Seine, études hydrographiques, Travaux souterrains de Paris; Dunod éd., Paris, tome 1, 88-96 
calcaires, à cause des fissures, les nappes sont discontinues et exposent à de graves mécomptes. Mais cette fâcheuse locution de nappe discontinue, faite de deux termes antinomiques (puisqu'une nappe ne peut être que continue),... s'excuse par la méconnaissance qu'on avait en 1872 de la vraie allure des rivières souterraines. Depuis lors, c'est par myriamètres qu'on les a trouvées et qu'on en a relevé des plans. Aussi. ne s'explique-t-on point l'erreur de ceux qui s'entêtent à ne pas les considérer comme la norme de la circulation en terrains fissurés.

Léon Janet, notamment, n'a jamais voulu se rendre à l'évidence et persista même toujours à employer, à dessein, le terme de nappe dans la craie (13)..."

\section{“HYPNOTISME DU CYCLE D'ÉVOLUTION MORPHOLOGIQUE (294-296)}

On s'est quelque peu laissé hypnotiser par le point de vue dit morphologique, qui a multiplié les complications, notamment en soulevant les questions d'âge évolutif.

Le travail posthume de Grund (14) admet ainsi quatre stades du paysage karstique : jeunesse (entonnoirs sporadiques), adolescence (chapelets de dolines), maturité (polje séparés par des crêtes), vieillesse (aplanissement ne laissant que peu de reliefs en saillie). Tout ceci est inspiré du cycle géographique de l'école américaine (15), qui a voulu catégoriser les développements et l'évolution des faits hydrogéologiques dans des formules et dénominations trop didactiques...

Ailleurs, on a prétendu qu'il y aurait trois stades successifs dans les régions caverneuses : Le premier (Jura) où le ruissellement n'a pas encore entraîné la terre végétale dans les gouffres qui demeurent absorbants. Le second (Causses) où la végétation n'est plus qu'au fond des entonnoirs. Le troisième (Karst) où le plateau est devenu complètement aride.

Ces distinctions sont illusoires; les reboisements du Karst ont bien réussi et fait renaïtre de larges espaces de végétation que le déboisement avait desséchés (16). Comme le Jura, il possède en même temps des abîmes morts et des gouffres actifs. Pour les Causses, leur aspect diffère uniquement selon l'altitude... Et Cvijic' est d'ailleurs arrivé (4) à énoncer qu' "il était bien difficile d'appliquer le cycle d'érosion de Davis au Karst parce que l'érosion karstique n'obéit pas aux mêmes lois que l'érosion normale"...

\section{"RÉSURGENCES SUSPENDUES (324)}

Il a pu arriver que certaines assises aquifères soient restées souterrainement si continues que l'érosion extérieure a creusé les vallées bien en-dessous d'elles; alors. on peut voir des résurgences sortir des flancs ou des falaises de ces vallées : elles sont demeurées suspendues bien au-dessus de leur ligne d'appel."

(13) JANET L. - 1900, Captage des sources des vallées du Loing et du Lunain, Livret guide du 8ème Congr. Géol. Intern. Paris

(14) GRUND A. - 1914, Der geographische Zyklus in Karst; Zeitschrift Gesellsch. für Erdkunde, Berlin, $\mathrm{n}^{\circ} 8,621-40$

(15) DAVIS W.M. - 1899, The Geographical Cycle; Geogr. Essays, 249-278

(16) DIMITZ L. - 1905, Die Förstlichen Verhältnisse u. Einrichtungen Bosniens und der Herzegovina, Wiss Mitt. B.H.N., Wien, VIII, 389 Goll und Putick - 1895, Die Karst Aufforschung in Krain, Laibach 


\section{$2 \%$ Creusement en régime noyé}

\section{LES ABÎMES}

Delagrave éd., Paris, 1894, 580 p.; pages 172, 531

"Au point de vue hydrologique, voici les observations faites au fond du Mas Raynal (fig. 2) :

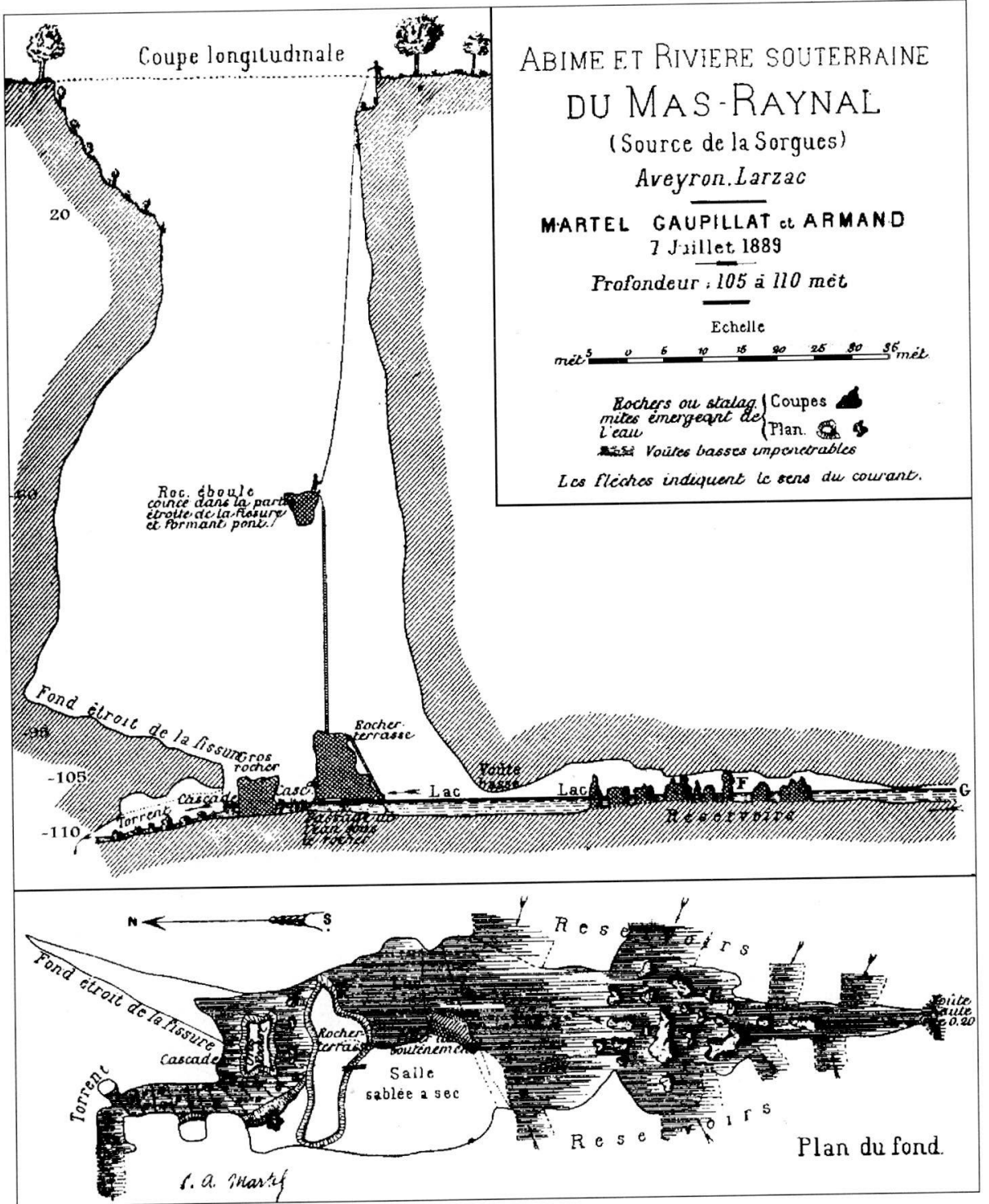

Fig. 2 : Le Mas Raynal (Aveyron) (Les Abîmes, P. 173). 
Sur les marnes horizontales (ou à peine inclinées) du Lias supérieur se cachait, sous la voûte basse presqu'à fleur d'eau des calcaires fissurés superposés, le réservoir naturel où commençait le torrent souterrain : la roche perméable (de suintement) formait un plafond inégal, dont les saillies reposaient par leurs pointes inférieures sur la formation imperméable; très larges étaient ces saillies, et d'une section certainement supérieure à la surface des vides, où l'eau s'étalait entre elles, avec une profondeur de 0,50 à 1 mètre à peine. - Si aux eaux très basses on pouvait pénétrer loin parmi ces vides, et en dresser le plan, ce plan aurait sans doute la figure d'un assemblage de pilastres irréguliers et plus épais que leurs intervalles."

En fait, au Mas Raynal, nous sommes tombés, au fond du gouffre, juste sur le point où s'opère la concentration de plusieurs galeries, presque sans pente, en une seule; elle s'effectuait dans un lac de 60 à 80 mètres de longueur tout au plus, et de 15 à 25 mètres de largeur, qu'on ne saurait appeler une nappe d'eau; au pourtour de la partie supérieure de ce lac, l'eau débouchait, par diverses fissures formant autant d'aqueducs, malheureusement trop peu élevés pour être suivis.

Au contact des terrains fissurés et des terrains imperméables, trois cas peuvent se présenter selon la figure que revêt la surface supérieure de la zone imperméable.

Premier cas - Si cette surface est horizontale, l'eau s'accumule et se disperse dans la partie inférieure des galeries ou conduites, plus ou moins larges, formées par les cassures plus ou moins grandes de la masse perméable superposée : en coupe verticale, on aura la disposition (fig. 3) de canaux séparés les uns des autres par des parois compactes; en plan, la figure horizontale sera celle d'un véritable delta, d'une carrière à plâtre, où on laisse subsister des piliers de soutènement, ou enfin d'un édifice à colonnes très irrégulier; avec cette différence que les vides occuperont assurément moins d'espace que les pleins, parce que les porte-à-faux, les voûtes trop vastes provoqueraient l'écroulement du système entier. Nous avons expliqué pourquoi, à propos du Mas Raynal qui est la réalisation absolue de ce cas, en réalité assez exceptionnel...

\section{Coupe transversale verticale}
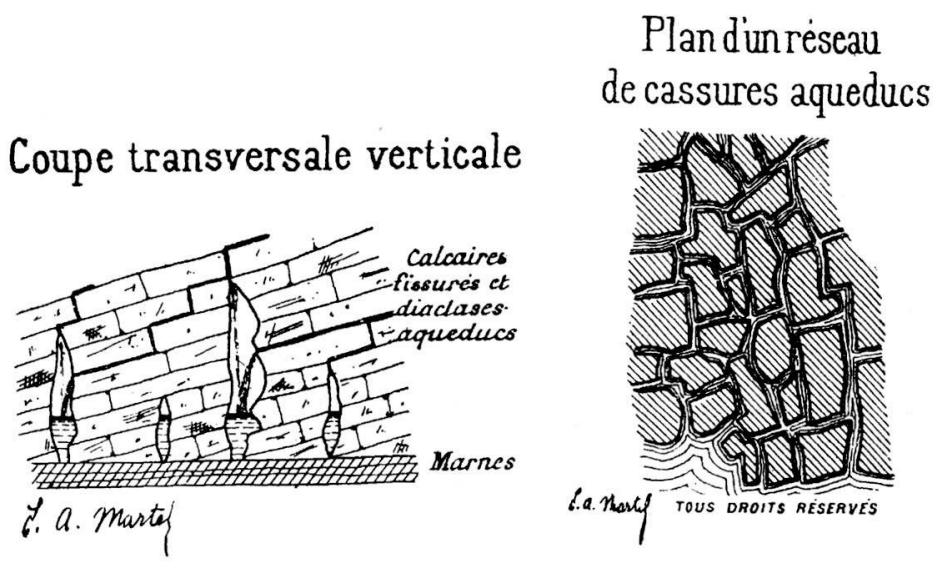

Fig. 3 : Schémas théoriques du cas où le calcaire repose sur une couche imperméable horizontale (Les Abîmes, p. 531), élévation et plan. 
Deuxième cas - Si la surface imperméable est en pente. il n'y aura de modifiée que la vitesse de l'écoulement qui se fera plus ou moins rapide selon le degré de l'inclinaison.

Troisième cas - Enfin, si la surface imperméable est en fond de bateau (fig. 4), se creuse en thalweg concave, il n'y aura pas de nappe non plus, car la gravité tendra à rassembler les eaux dans les cassures les plus basses, les plus rapprochées de la concavité; ces cassures inférieures verront le liquide s'élever plus haut le long de leurs parois, y présenter plus de profondeur, y exercer une érosion plus vive que dans les cassures supérieures, lesquelles seront plus fréquemment dépourvues d'eau.

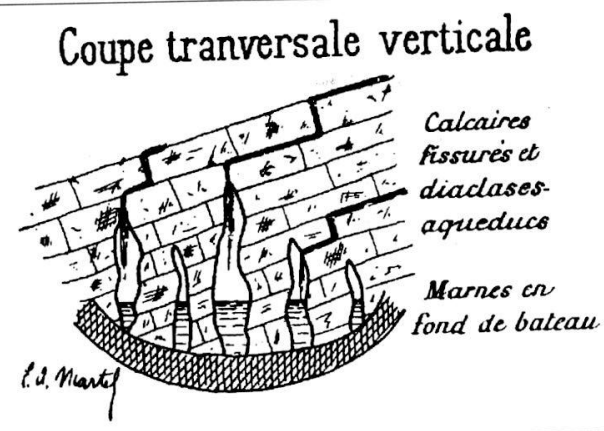

Fig. 4: Schéma théorique du cas où le calcaire repose sur une couche imperméable en fond de bateau (Les Abîmes p. 531), élévation.

\section{IRLANDE ET CAVERNES ANGLAISES}

Delagrave éd., Paris, 1897, pages 102-4, 177-181

J'ai relevé sur la rive méridionale du Lough-Mask... des effets de corrosion tels que je n'en ai remarqué nulle part (17); la grève est couverte de gros blocs de calcaire carbonifère noir, dont toutes les faces, creusées de cupules profondes de 5 à 10 centimètres, semblent de véritables écumoires (photos a et b) : il faut que les pluies (ou les hautes eaux du lac) soient singulièrement chargées d'acide carbonique pour ronger à ce point une pierre si dure. Et si l'eau du Lough-Mask... a mangé la roche de pareille façon, il est très possible que la corrosion seule ait frayé un passage au liquide [souterrainement vers le Lough-Corrib, faisant de la zone intermédiaire] une réelle éponge à larges pores, sans que l'érosion ait eu besoin d'y façonner les joints et les diaclases en ces longues et larges galeries que nous sommes habitués à rencontrer partout sous les terrains calcaires. L'eau souterraine s'y propage probablement en tous sens, entre les parallélipipèdes de la roche...

(17) lough = lac (irlandais) des formes de corrosion analogues [qui furent attribuées à la décomposition acide des mousses et lichens] existent sur les bords du Lough-Corrib [distant de $4 \mathrm{~km}$ du Lough-Mask, et recevant ses eaux par une circulation souterraine dont la pente est de 2 pour 1000] 


\section{LA CAVERNE DE MITCHELSTOWN}

À l'intérieur, les galeries présentent deux aspects différents : les unes. les plus larges, ont servi et servent encore de points d'absorption aux eaux du dehors...; les autres fissures, généralement plus étroites et situées dans les parties inférieures de la caverne, ont conduit ces eaux on ne sait où : soit à quelque source lointaine indéterminée, soit même dans les profondeurs de l'écorce terrestre; elles sont rendues impénétrables, tantôt par des éboulis de pierres,... tantôt par l'étroitesse des crevasses, de plus en plus resserrées dans la portion sud de la caverne; cette dernière disposition rappelle exactement celle de la grande grotte du Cro de Grandville ou de Miremont dans la Dordogne,... et l'on peut se demander si, comme celle-ci, la caverne de Mitchelstown n'a pas servi à vider par le fond quelque grand lac d'une ancienne époque géologique...

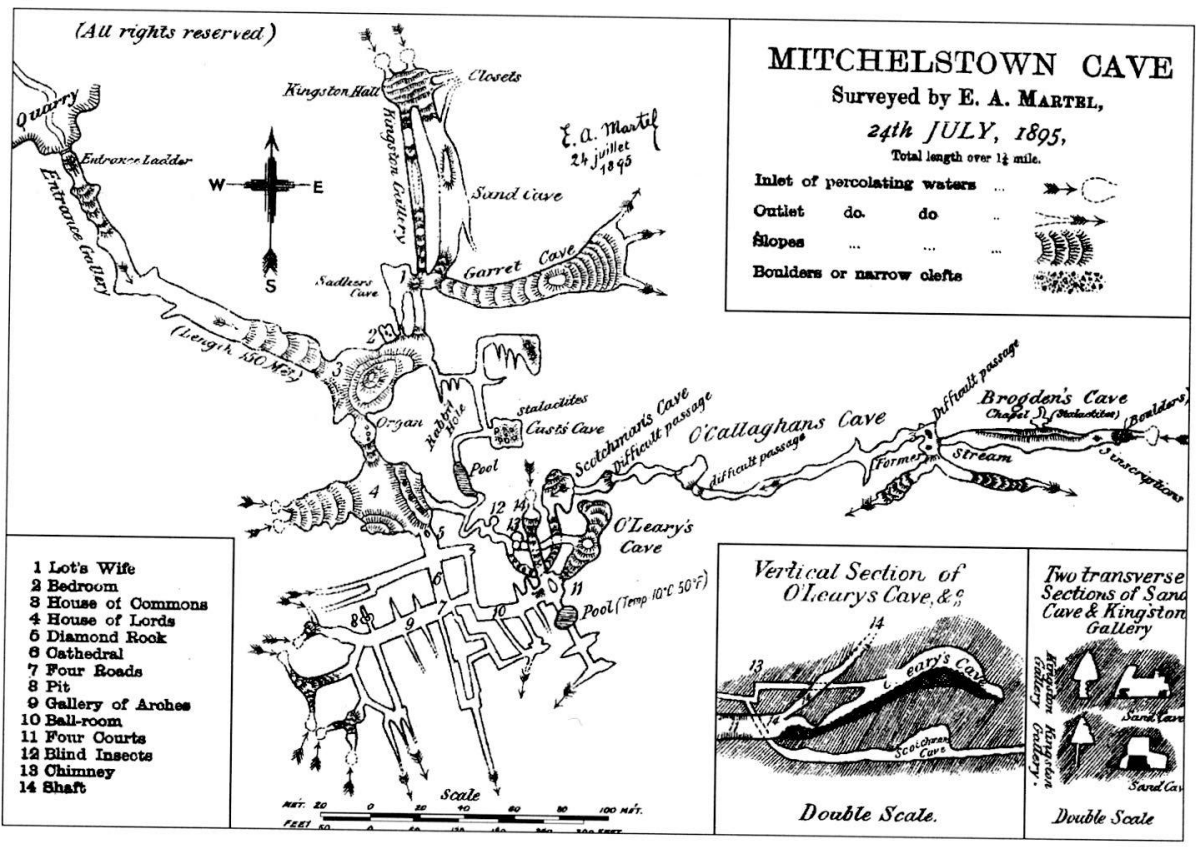

Fig. 5 : Michelstown cave, en Irlande (Irlande et cavernes anglaises, planche hors texte)

La disposition en damier des diaclases est remarquable dans la portion sud (voir le plan) : trois jeux de fissures perpendiculaires entre eux ont découpé là de grands polyèdres de roche, souvent tout à fait cubiques, dont les interstices à angles droits ont laissé fuir les eaux qui les élargissaient; en profondeur, ils se resserrent d'autant plus qu'ils se ramifient davantage... Le terrain... est le même (calcaire carbonifère) [qu'entre le Lough-Mask et le Lough-Corrib] où, comme je l'ai indiqué, les eaux actuelles doivent probablement circuler dans un réseau de crevasses de ce genre. On s'explique que les galeries d'absorption soient presque toutes... dans la partie sud de la grotte, quand on remarque que telle est la direction générale du pendage (à $40^{\circ}$ ) des strates calcaires. 


\section{LA GROTTE DE LA BALME (ISÈRE)}

Mém. Soc. Spéléologie, III/19, 1899, page 15

Jadis, avant la brusque ouverture de la caverne, alors que le pan de muraille la séparant de la vallée du Rhône n'était pas encore abattu, la Balme devait être remplie d'eau jusqu'aux voûtes (on a constaté que, en amont des siphons reconnus, cela se produisait souvent pendant les crues dans les cavernes de la Recca, en Istrie...); en plusieurs endroits, on distingue et on parcourt partiellement dans [ces voûtes] des fissures verticales plus ou moins étroites, vraies cheminées intérieures (labyrinthe du Nord ou de Mandrin, labyrinthe du Sud...), qui formaient la tuyauterie interne du plateau et y amenaient. par de nombreuses conduites, les eaux d'infiltration. C'est dans cette tuyauterie, nous venons de le voir, que les eaux se mettaient en pression.

\section{LES CAVERNES ET LES RIVIÈRES SOUTERRAINES DE LA BELGIQUE}

par E. Van den Broeck, E.A. Martel \& Ed. Rahir, II, 1910, pages 1534-1535

Avec les sorties d'eau du Calcaire carbonifère [du bassin de Dinant] apparaït un élément nouveau, ou tout au moins inhabituel dans nos explorations des calcaires dévoniens, savoir : les Résurgences sourcières, caractérisées par leur double origine, et dans lesquelles, à l'apport permanent d'eaux élaborée, donc potables, vient s'ajouter un afflux généralement temporaire d'eaux brutes superficielles...

Mais ce qui différencie complètement les calcaires carbonifériens des calcaires dévoniens, c'est la fréquente présence de sources véritables et permanentes, d'eaux parfaitement filtrées et élaborées, émergeant spécialement du Calcaire tournaisien.

\section{NOUVEAU TRAITÉ DES EAUX SOUTERRAINES}

Comme l'absolu n'existe pas -, il est indispensable de reconnaître que certaines dispositions tectoniques... peuvent attirer parfois de puissants amas d'eaux dans le fond de synclinaux très aigus... Mais ce sont alors des poches ou amas d'eau limités latéralement, plus semblables à des citernes qu'à des nappes, dont ils ne donnent que l'illusion. Ils sont généralement statiques, sans mouvement, fossiles même par places. 


\section{LA FRANCE IGNORÉE (DES ARDENNES AUX PYRÉNÉES)}

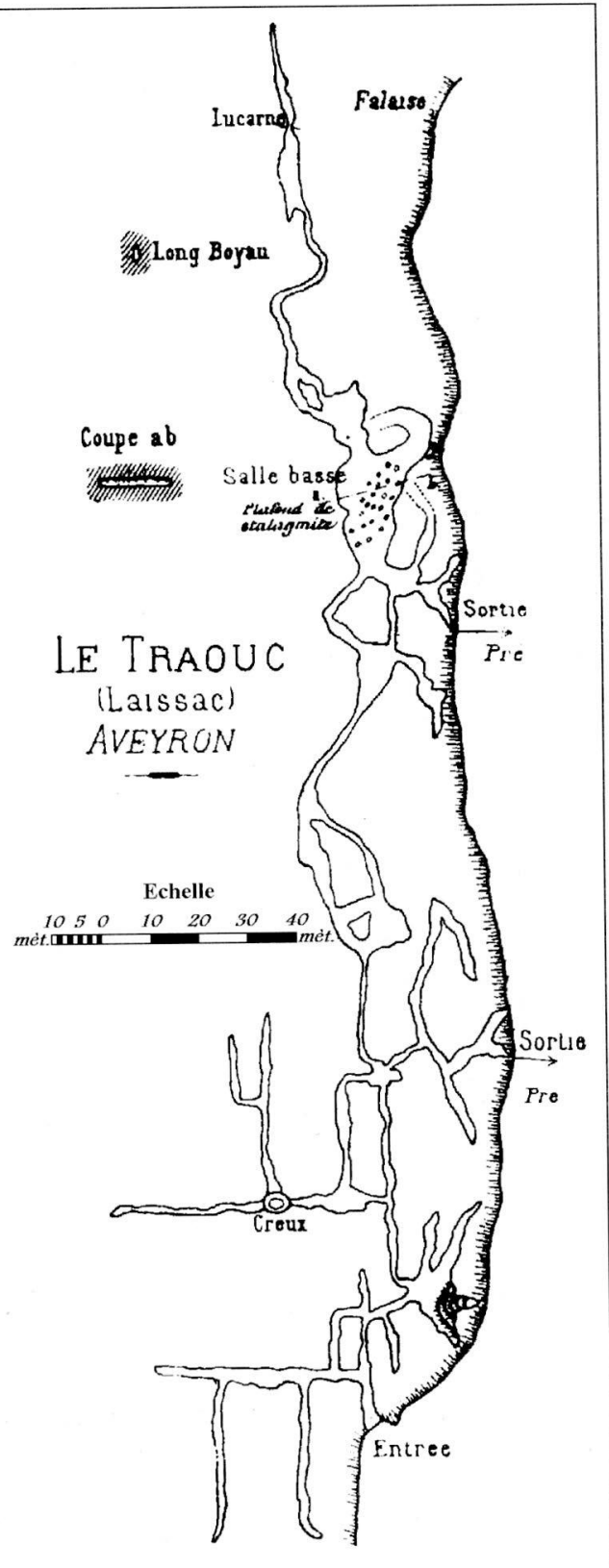

Delagrave éd., Paris, 1930, pages 110-111

A Laissac, la grotte du Traouc (le Trou) est un labyrinthe qui montre à merveille comment les eaux souterraines ont utilisé les cassures du sol pour excaver les cavernes. Le plan de Gaupillat (juillet 1892) a mesuré $850 \mathrm{~m}$ de galeries.

Quelques autres ont été trouvées depuis, et beaucoup d'amorces de couloirs sont bouchées. Jadis le ruisseau de Mayroux (forêt des Palanges) avait ici des pertes... Ses eaux souterraines ont pratiqué le damier irrégulier du Traouc; les voûtes sont basses et les sections étroites; plusieurs galeries aboutissent au dehors. Les grottes de Mitchelstown (Irlande) en calcaires carbonifères, de Sloup en Moravie, près de Brünn (18) en calcaires dévoniens, etc... présentent exactement cette même disposition.

Fig. 6 : Le Traouc, à Laissac (Aveyron), d'après Gabriel Gaupillat (La France Ignorée p. 111)

(18) = Brno (Moravie, République tchèque) 


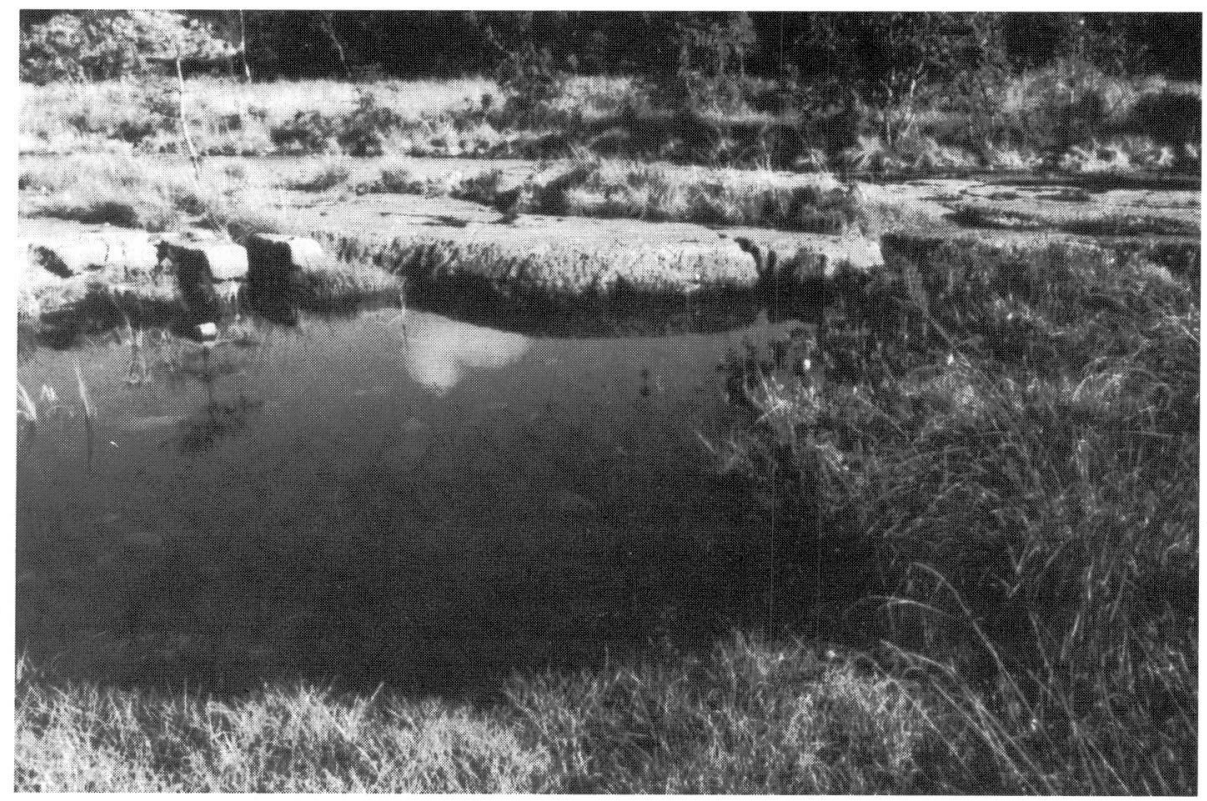

photo a (cliché CHOPPY) : site du Loug-Mask

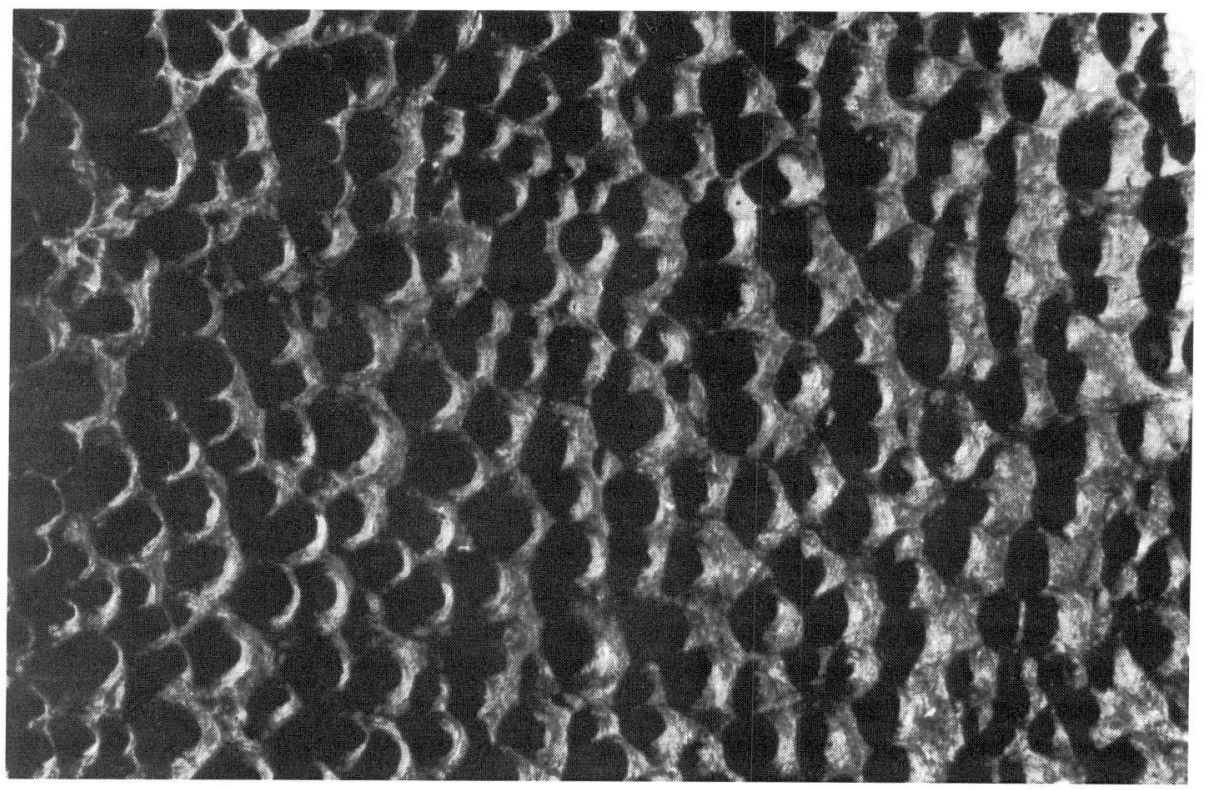

photo b (cliché CHOPPY) : les petites alvéoles du Lough Mask, d'environ 2 centimètres de diamètre; de loin en loin, il existe d'autres alvéoles, ascendantes, toujours semi cylindriques, de 8 à 10 centimètres de diamètre 